\title{
Extralobar Supradiaphragmatic Pulmonary Sequestration Arising from the Retroperitoneum Through a Congenital Diaphragmatic Defect
}

\author{
Soojin Lee, M.D., Jeong Su Cho, M.D., Hoseok I, M.D., Hyo Yeong Ahn, M.D., Yeong Dae Kim, M.D. \\ Department of Thoracic and Cardiovascular Surgery, Pusan National University Hospital, Pusan National University School of Medicine, Busan, Korea
}

\section{ARTICLE INFO}

Received June 9, 2020

Revised August 26, 2020

Accepted August 29, 2020

Corresponding author

Jeong Su Cho

Tel 82-51-240-726

Fax 82-51-243-9389

E-mail pnuhts@gmail.com

ORCID

https://orcid.org/0000-0002-7422-6171
Here, we report the rare case of a 13-year-old girl with a congenital diaphragmatic hernia (also known as Bochdalek hernia), which was revealed to be an extralobar pulmonary sequestration that was treated using laparoscopic and video-assisted thoracic surgery sequestrectomy and repair of the diaphragm defect after detection of a supradiaphragmatic mass connected with the retroperitoneum. The patient showed no postoperative complications at a 1-month follow-up examination.

Keywords: Bronchopulmonary sequestration, Congenital diaphragmatic hernias, Videoassisted thoracic surgery

\section{Case report}

A 13-year-old girl with no medical history was admitted for evaluation of repeated upper abdominal pain, which had started 3 months before presentation. No abnormal findings were reported, except a small congenital diaphragmatic hernia $(\mathrm{CDH})$ (also known as a Bochdalek hernia). The hernia was noted on the left side on enhanced chest computed tomography (CT) (Fig. 1).

We planned to repair the defect through video-assisted thoracic surgery (VATS) exploration rather than regular imaging follow-up owing to the risk of herniation of abdominal contents into the thoracic cavity, which might result in strangulation and incarceration. The patient was placed in the right lateral decubitus position. After a thoracoscopic trocar port and 2 other instrument ports were placed, a 4-cm-wide diaphragm defect with a consolidative mass was found overlying the left diaphragm from the end of the upper pole of the spleen, beneath the diaphragm. The pinkish mass covered by its own capsule could hardly be differentiated from the spleen, liver, or adrenal gland (Fig. 2A). We decided to perform a laparoscopic exploration to identify the origin of the mass, owing to the limited view in the thoracoscopic approach. After the patient's po- sition was changed from the right lateral decubitus to the semi-lateral position, with the abdomen facing upward, 3 laparoscopic ports were placed. There was no intraperitoneal structure originating to the thoracic cavity, and mobilization of the spleen and fundus was performed to check the overall diaphragm (Fig. 2B) Moreover, a vessel bundle that drained into the splenic artery and splenic vein, respectively, coursing into the thoracic cavity through the diaphragmatic defect was identified. The artery and the stalk of the mass were separated by $10-\mathrm{mm}$ Hem-o-lok (Teleflex, Wayne, PA, USA) double clipping, followed by primary and reinforcement sutures of the diaphragmatic defect with Prolene 2-0 and silk 2-0 sutures (Fig. 3). The specimen, which measured $4.5 \times 4.0 \times 2.0 \mathrm{~cm}$, was extracted, and the pathology report indicated that the bronchus was identified on the the center of the resected sample. The histopathologic examination was consistent with the diagnosis of extralobar pulmonary sequestration (EPS) (Fig. 2C).

The patient was discharged on postoperative day 2 without any abnormalities in laboratory results or chest radiographs. In serial follow-up at the outpatient department, she did not report any respiratory discomfort or abdominal pain, which was the initial symptom at the time of admission. 

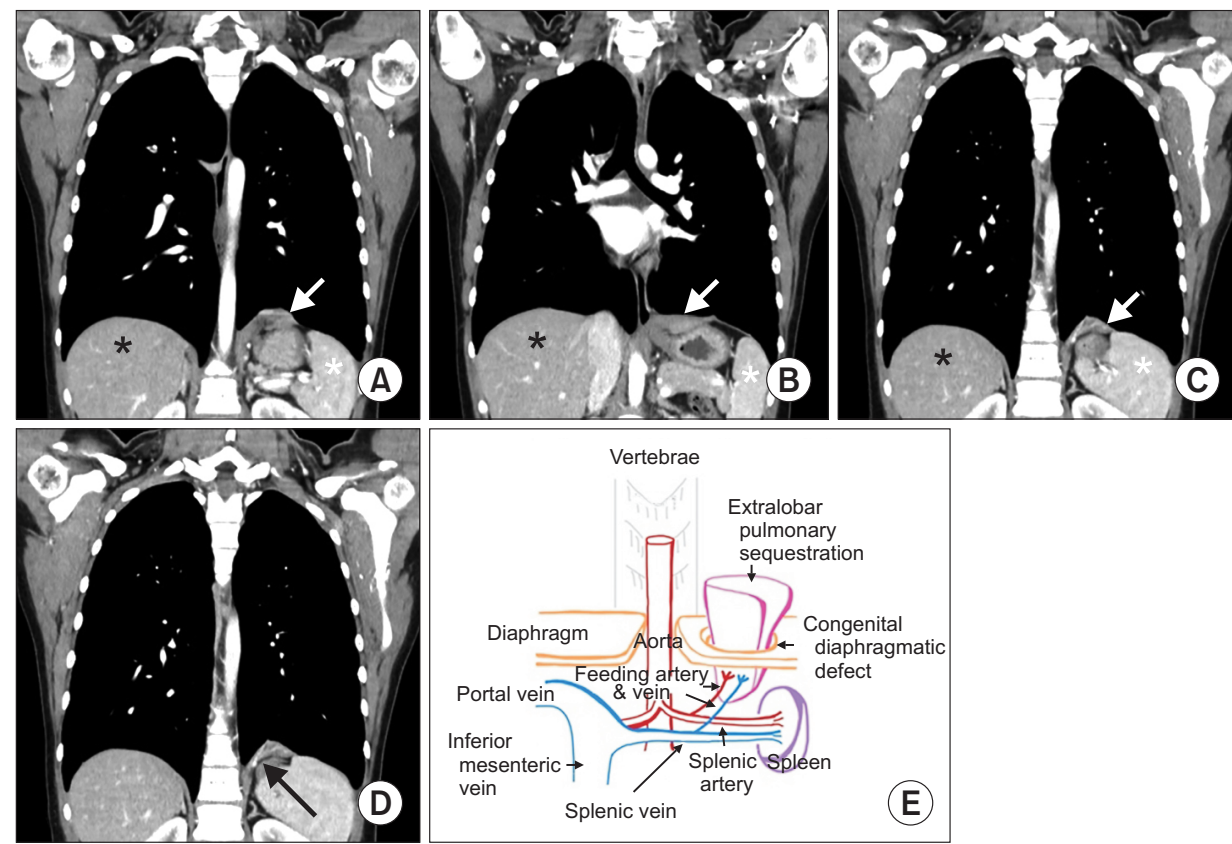

Fig. 1. Initial enhanced chest computed tomography showing a left-sided Bochdalek hernia. (A) Extralobar pulmonary sequestration, which was identified as herniated omentum on the initial reading. (B) View of the extralobar pulmonary sequestration close to the left lobe of the liver, which was difficult to distinguish from liver herniation. (C) View of the extralobar pulmonary sequestration close to the spleen, which was difficult to distinguish from spleen herniation. (D) View showing the diaphragmatic defect. (E) Diagram illustrating the cross-sectional anatomy of the extralobar pulmonary sequestration arising from the retroperitoneum through the diaphragmatic defect, with arterial supply from the splenic artery and venous return to the splenic vein (black asterisk: liver, white asterisk: spleen, white arrow: supradiaphragmatic mass, black arrow: diaphragm discontinuity with the bundle of the feeding artery and vein of the extralobar pulmonary sequestration).

The study was approved by the institutional review board of Pusan National University Hospital (IRB approval no., 2011-011-097). The patient provided written informed consent for publication of the research details and clinical images.

\section{Discussion}

Pulmonary sequestration is a developmental anomaly consisting of a non-functioning lung supplied by more than 1 systemic artery. Its venous drainage is into the azygos or hemi-azygos system, and it may or may not communicate with the bronchial tree. Depending on the location of the malformation within the normal pulmonary parenchyma, it is classified as intralobar or extralobar, in which the pulmonary sequestration is situated within or separate from the normal lung parenchyma and covered by its own pleura, respectively [1].

The incidence of pulmonary sequestration is estimated to be $0.15 \%-1.7 \%$ [2]. EPS is relatively uncommon, accounting for $25 \%$ of pulmonary sequestrations [3]. EPS is most frequently intrathoracic and is frequently found on the left side. EPS is rarely found intra-abdominally, and most such cases have been reported near the diaphragm [4]. In our case, it is remarkable that EPS was found neither within the intrathoracic or intra-abdominal cavity, but was initially detected in the supradiaphragmatic area and was revealed to be located in the arch extending from the retroperitoneal space, where it has seldom been reported (Fig. $1 \mathrm{E})$.

The extralobar type results from a more complex embryonic defect than the intralobar type, and the associated anomalies are more frequent and serious. The prevalence of EPS associated with $\mathrm{CDH}$ on the left side is higher than that of EPS associated with other associated malformations [5]. Other studies have not reported proportions of cases comparable to that described in our study, as this specific combination of features appears to occur too rarely to count. Most cases of EPS are located close to the diaphragm. It is challenging to distinguish EPS from other malformations or herniated intra-abdominal organs, such as ectopic liver, suprarenal neuroblastoma, teratoma, foregut duplication, and cystic adenomatoid malformation [6]. The differential diagnosis may be possible using preopera- 

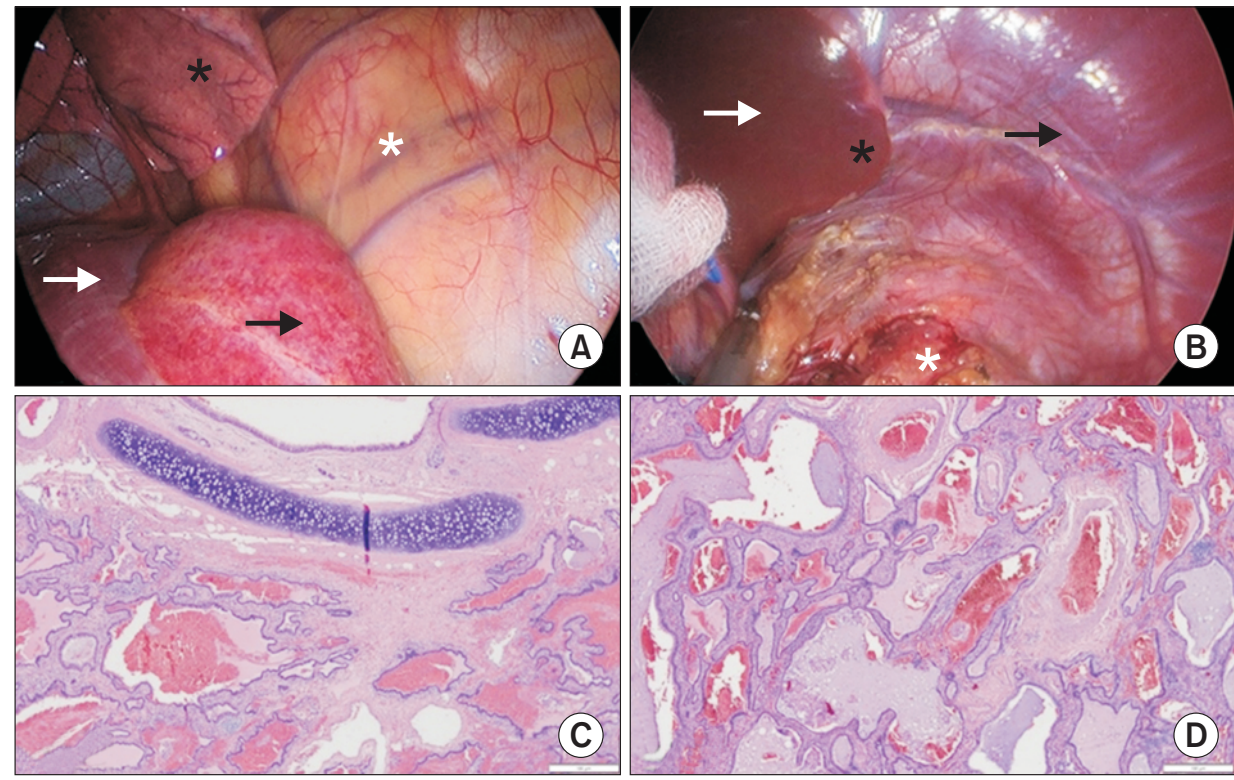

Fig. 2. (A) Thoracoscopic findings. The extralobar pulmonary sequestration measuring $4.5 \times 4.0 \times 2.0 \mathrm{~cm}$ was laid over the left diaphragm (black asterisk: lower lobe of the left lung, white asterisk: vertebrae, black arrow: extralobar pulmonary sequestration, white arrow: diaphragm). (B) Laparoscopic findings: absence of an intraperitoneal structure connecting with the extralobar pulmonary sequestration through the diaphragmatic defect (black asterisk: location of extralobar pulmonary sequestration, which is not seen in this view, through the diaphragmatic defect covered by the left lobe of the liver; white asterisk: retroperitoneum; black arrow: diaphragm). (C, D) Microscopic findings of resected extralobar pulmonary sequestration (H\&E staining, $\times 40$ magnification). (A) The peripheral area of the resected specimen shows bronchial cartilage and bronchial epithelium-lined space. (B) The center of the tissue was consistent with sequestrated lung tissue. It showed poorly developed alveoli or alveolar ducts and resembled dilated bronchiole structures.

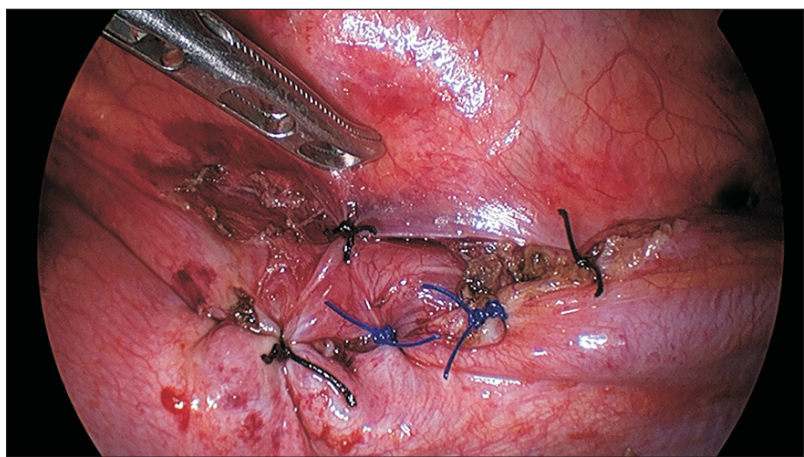

Fig. 3. Thoracoscopic view of the sutured diaphragmatic defect.

tive ultrasonography; however, a definitive diagnosis is achieved only by angiography [1]. In this patient, the mass was indistinguishable from other associated malformations of $\mathrm{CDH}$ or herniated intra-abdominal organs because the thoracoscopic view was limited to the supradiaphragmatic plane. Laparoscopic exploration confirmed that there was no communication with abdominal organs or vessels that would need to be removed.

The diagnosis of $\mathrm{CDH}$ can be confirmed by ultrasonography, based on the signs of an uninterrupted contour of the diaphragm and bowel peristalsis in the thorax. Contrast abdominal CT has high specificity; however, it cannot be used as a definitive tool for diagnosis, because it is unable to image diaphragmatic defects that are located in different scanning planes [7]. Preoperative abdominal CT showed only a small, left-sided CDH (also known as a Bochdalek hernia) with a partially herniated omentum, which resulted in the decision to repair the diaphragmatic hernia using the thoracoscopic approach. On visual inspection, a wedge-shaped solid organ was detected, and it could be easily confused with other intraperitoneal solid organs, such as the spleen and liver. $\mathrm{CDH}$ associated with an EPS in a rare location poses considerable diagnostic challenges, often resulting in inappropriate treatment with a potentially fatal outcome. Incorrect recognition and resection of the intraperitoneal organ without a further evaluation of the herniated organ for differential diagnosis may lead to massive bleeding, post-hepatectomy liver failure, and bile leakage after partial liver resection. Furthermore, venous thromboembolism and post-splenectomy infection resulting from splenectomy or iatrogenic perforation of the stomach are potential complications [8].

In this case, preoperative chest CT had limitations for 
differentiating EPS with a herniated intra-abdominal organ. The initial plan included simply repositioning the herniated omentum, followed by repair of the diaphragmatic hernia by VATS exploration. Although VATS inspection suggested the need for a further evaluation of the unknown mass, a laparoscopic exploration made it clear that the mass was an EPS arising from the retroperitoneum through a $\mathrm{CDH}$. Considering the extremely unusual presentation of EPS and the limitations of CT findings, additional laparoscopic exploration may be required for an underevaluated, unknown organ found in the supradiaphragmatic plane, especially associated with $\mathrm{CDH}$. We share this rare case to aid other clinicians in making cautious decisions regarding the management of these patients.

\section{Conflict of interest}

No potential conflict of interest relevant to this article was reported.

\section{Acknowledgments}

This work was supported by a clinical research grant from Pusan National University Hospital in 2020.

\section{ORCID}

Soojin Lee: https://orcid.org/0000-0003-4464-1451

Jeong Su Cho: https://orcid.org/0000-0002-7422-6171
Hoseok I: https://orcid.org/0000-0001-8930-8148

Hyo Yeong Ahn: https://orcid.org/0000-0003-3198-8237

Yeong Dae Kim: https://orcid.org/0000-0001-7135-4648

\section{References}

1. Carter R. Pulmonary sequestration. Ann Thorac Surg 1969;7:68-88.

2. Skandalakis JE, Gray SW, Symbas P. Pulmonary circulation. In: Skandalakis JE, Gray SW, editors. Embryology for surgeons: the embryological basis for the treatment of congenital anomalies. Baltimore (MD): Williams \& Wilkins; 1994. p. 463-70.

3. Kocaoglu M, Frush DP, Ugurel MS, Somuncu I. Bronchopulmonary foregut malformations presenting as mass lesions in children: spectrum of imaging findings. Diagn Interv Radiol 2010;16:153-61.

4. Kalenahalli KV, Garg N, Goolahally LN, Reddy SP, Iyengar J. Infradiaphragmatic extralobar pulmonary sequestration: masquerading as suprarenal mass. J Clin Neonatol 2013;2:146-8.

5. Valle AR, White ML Jr. Subdiaphragmatic aberrant pulmonary tissue (case report). Dis Chest 1947;13:63-8.

6. Morad NA, al-Malki T, e-Tahir M. Intra-abdominal pulmonary sequestration: diagnostic difficulties. Pathology 1997;29:218-20.

7. Heiberg E, Wolverson MK, Hurd RN, Jagannadharao B, Sundaram M. CT recognition of traumatic rupture of the diaphragm. AJR Am J Roentgenol 1980;135:369-72.

8. Kajal P, Bhutani N, Goyal M, Kamboj P. Iatrogenic gastric perforation in a misdiagnosed case of late presenting congenital diaphragmatic hernia: report of an avoidable complication. Int J Surg Case Rep 2017;41:154-7. 